\title{
Kimura's Disease: A Rare Cause of Postauricular Swelling
}

\author{
Suman Kumar Das, ${ }^{1}$ Mukesh Kumar Singh, ${ }^{1}$ Indranath Kundu, ${ }^{1}$ Swapan Kumar Ghosh ${ }^{1}$
}

Introduction

\section{$\underline{\text { ABSTRACT }}$}

Kimura's Disease is a chronic inflammatory disorder of lymph node which is very rare in Indian population.

Case Report

A 15 year old boy with multiple postauricular swelling for 18 months presenting in OPD and diagnosed having eosinophilia.

Then excision biopsy was taken, which indicates Kimura's Disease. Patient was treated with high dose of corticosteroid.

Conclusion

Kimura's disease, though rare should be kept in mind for treating a patient with lymphadenopathy with eosinophilia or high IgE

level, because it can spare the patient unnecessary invasive procedure.

$\underline{\text { Keywords }}$

Kimura Disease; Eosinophilic Lymphadenitis; Lymphadenopathy

$\mathrm{K}$ imura's disease is a rare, benign, chronic inflammatory disorder of unknown aetiology that involves lymph nodes and subcutaneous tissue of head and neck region. The condition is clinically characterized by triad of painless subcutaneous masses, blood and tissue eosinophilia and markedly increased serum IgE concentrations. The disorder is primarily seen in East Asian male patients in the third decade of life, though rare in Indian. Although it may mimic a neoplastic condition, early correct diagnosis of Kimura's disease may spare the patient from unnecessary invasive diagnostic procedure. We present such a case in a 15 year old boy who presented with bilateral postauricular swelling and focus on treatment modalities in our discussion.

\section{Case Report}

A 15 year old boy was referred to our outpatient department for workup for multiple bilateral postauricular subcutaneous swelling for 18 months. He was seen by his primary care physician who treated him with different types of oral antibiotics for many weeks. There was no history of fever, sore throat, cough, ear discharge or weight loss. Physical examinations showed an apparently well boy with multiple distinct palpable masses at the both postauricular areas which were nontender, firm, non-fluctuant and freely mobile (Fig. 1). The largest discrete mass was $2.5 \mathrm{~cm}$ in diameter. The rest of the general physical and systemic examinations were normal.

Peripheral blood smear showed $11 \%$ eosinophils with absolute eosinophil count of 950/cumm. Chest radiograph was within normal limits. Tuberculosis skin test and rapid Infectious mononucleosis assay were negative.

Because of concern that the mass might be some lymphoproliferative disorder or neoplasm, an excision biopsy was taken. Histopathological examination of the mass showed eosinophilic infiltration and vascular proliferation with prominent endothelial cells within the extra nodal soft tissue associated with lymphoid hyperplasia and germinal centre formation. Serum IgE levels were estimated which showed - $5300 \mathrm{KIU} / \mathrm{ml}$ (Normal - $150 \mathrm{KIU} / \mathrm{ml}$ ) further supported the diagnosis of Kimura's Disease.

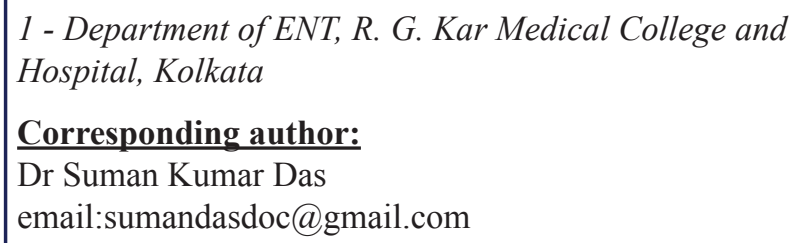




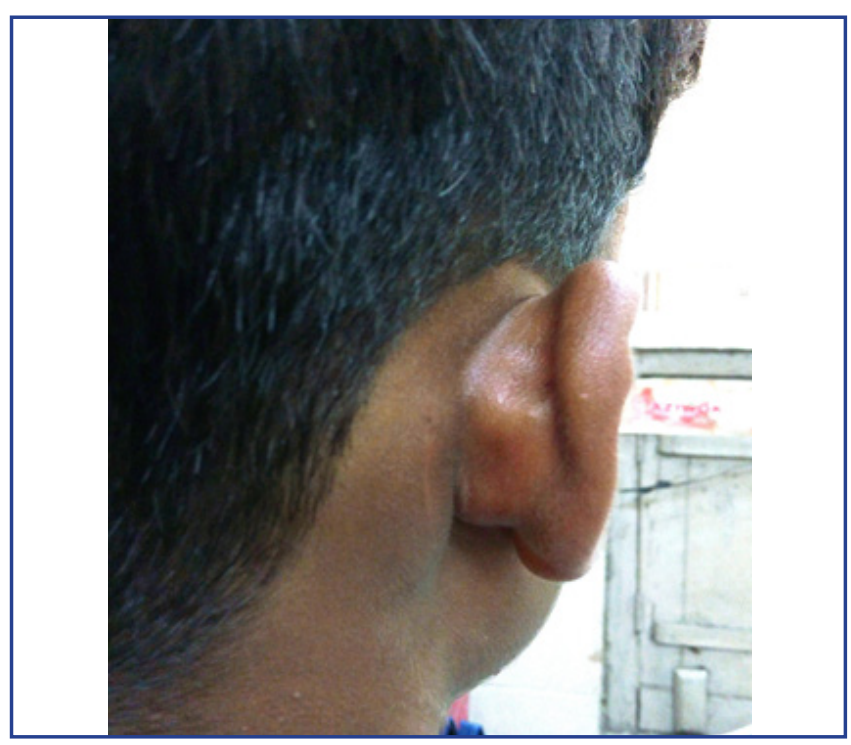

Fig.1. Postauricular swelling of a 15 year old boy

After 6 months follow up, the mass began to recur and then treatment with oral prednisolone was started at a dose of 40mg/day for the initial period of 6 week and subsequently tapered every week by $10 \mathrm{mg} /$ day over next 4 weeks. After 4 weeks of treatment eosinophilia was decreased and became well within normal limits in 6 weeks. IgE levels fell to $144 \mathrm{KIU} / \mathrm{ml}$ gradually. There was no local recurrence at 9 months follow up.

\section{Discussion}

Kimura's Disease was first described by Kim and Szeto ${ }^{1}$ in Chinese literature in 1937, but was later characterized by Kimura et al in 1948 in a manuscript titled "On the unusual granulation combined with hyperplastic changes of lymphatic tissue. ${ }^{2}$ It is now recognized as a benign cause of painless localized lymphadenopathy of Asians, especially boys of Chinese and Japanese origin. ${ }^{3}$ The clinical triad of subcutaneous nodule in head and neck region, prominent peripheral eosinophilia and highly elevated IgE, particularly when seen in an Asian male [in 2nd and 3rd decades of life, although can become apparent at any age] is highly suggestive of Kimura's Disease.

The clinical course of Kimura's Disease is benign and self-limited. The subcutaneous masses or adenopathy are usually found in head and neck region with occasional pruritus of overlying skin. It sometimes affects axillary, periauricular, inguinal or epitrochlear node. ${ }^{4}$ Most patients have a prolonged course with slow enlargement of masses. Occasional spontaneous resolution is known. These lesions do not have any malignant potential. Kimura's Disease may be complicated by renal involvement. In case of renal involvement, nephrotic syndrome is the commonest presentation, proteinuria may occur in $12-16 \%$ of cases. ${ }^{5}$ But in our case, there was normal renal function and there was no evidence of proteinuria.

The pathophysiology of Kimura's Disease is not well understood at this time, but may relate to a disturbance in the normal rate of product of an interaction between types 1 and 2 helper cells. Such a derangement could result in excessive elaboration of eosinophilotrophic cytokines. ${ }^{6}$ These patients have been shown to have high levels of circulating eosinophilic cationic protein and major basic protein with heavy concentration of IgE in their tissues. ${ }^{7}$ Allergic or parasitic etiologies for Kimura's Disease have been actively sought, but not proven.

The pathology of Kimura's Disease is characterized by prominent germinal centres in involved lymph nodes containing cellular, vascular and fibrous components. The cellular component consists of dense eosinophilic infiltrate in background of abundant lymphocytes and plasma cells, eosinophilic micro-abscesses with central necrosis. ${ }^{8,9}$ Other characteristics may include sclerosis, proteinaceous material and / or vascularity in the germinal centres and polykaryocytes of WarthinFinkeldely type. ${ }^{10}$

The lesions can be further investigated with ultrasonography, CT scan and MRI. But their radiographic features are not pathognomonic. The diagnosis is made only by histopathological examination of excisional biopsy specimen of lesion.

The differential diagnosis of Kimura's Disease includes other entities such as Tuberculous adenitis, Mikulicz's Disease, Eosinophilic Granuloma, ALHE (Angiolymphoid Hyperplasia with Eosinophilia), Cylindroma, Pyogenic granuloma. It should also mimic some serious disorder like Hodgkin's Disease, Acute non-lymphocytic leukemia, follicular lymphoma 
and Kaposi's sarcoma. Except for Angiolymphoid Hyperplasia with Eosinophilia, the clinical and histological features of these diseases easily distinguish them from Kimura's Disease.

There is no consensus on management of Kimura's Disease. Various treatment modalities have been tried with variable success. Three major therapeutic options exist for Kimura's Disease. Excision of the mass remains the treatment of choice if the entire lesion can be removed, but local recurrence is common. Localized initial regrowth can often be managed with repeated surgical excision. Local irradiation has occasionally been used to treat recurrent, persistent or refractory cases to surgical and medical therapy, recalcitrant and large lesions, young patient or when surgery is not feasible. ${ }^{11}$ Finally, the pharmacotherapy of Kimura's Disease has mainly involved the use of systemic and intralesional corticosteroids for an adequate period have been shown to reduce the size of the lesion but the lesion tends to recur when the drugs are discontinued. ${ }^{11,12}$ Cyclosporine, oral pentoxifylline, all trans retinoic acid with prednisolone, imatinib, leflunomide, azathioprine and vincristine have been tried in the management for Kimura's Disease with variable responses. ${ }^{13}$

The choice of treatment modalities should be individualized. Recurrence is common with all the modalities of treatment.

Our patient had excision of postauricular mass but after 6 months, recurrence of mass happened. Then the patient was treated with $1 \mathrm{mg} / \mathrm{kg} /$ day of oral prednisolone for 6 weeks which allowed gradual regression of mass. The dose is then tapered over next 4 weeks.

\section{Conclusion}

Kimura's Disease may be suspected when eosinophilia or high IgE level are seen in a patient of slowly progressive head and neck swelling or lymphadenopathy.

If not properly diagnosed, the neck mass or postauricular mass may be initially mistaken for a malignancy leading to unnecessary and potentially invasive investigation. However due to a well-obtained clinical history and histological awareness a proper diagnosis has been established in our case.

\section{Acknowledgments}

We thank Dr. Anadi Roychoudhury, Associate Professor, Department of Pathology, for helping us with histopathological diagnosis of Kimura's Disease.

\section{References}

1. Viswanatha B. Kimura's Disease in children: A 9 years' prospective study. Int. J Pediatric Otorhinolaryngol. 2007; $71: 1521-5$

2. Kimura T, Yoshimura S, Ishikawa E. On the unusual granulation combined with hyperplastic changes of lymphatic tissue. Trans Soc Patho Jpn. 1948; 37:179-80

3. Motoi M, Norie Y, A Kagi T. Kimura's Disease: clinical, histological and immunohistochemical studies. Acta Med Okayama. 1992; 46: 449-55

4. Som PM, Biller NF. Kimura's Disease involving parotid gland and cervical nodes: CT scan and MRI findings. J Comput Assist. Omogz. 1992; 16:320-2

5. Atar S, Oberman AS, Flatou E et al. Recurrent nephrotic syndrome associated with Kimura's Disease in a young nonoriental male. Nephron 1994; 68: 259-61

6. Wierenga EA, Backx B, Snoek M et al. relative contributions of human type 1 and 2 T-helper cell derived eosinophilotrophic cytokines to development of eosinophilia. Blood 1993;82: 1471-9

7. Motoi M, Horie Y, A Kagi T. Kimura's Disease: Clinical, histological and immunohistochemical studies. Acta Med Okayama. 1992; 46: 449-55

8. Chong WS, Thomas A, Goh CL. Kimura's Disease and Angiolymphoid Hyperplasia with Eosinophilia: Two disease entities in the same patient: Case report and review of the literature. Int. J Dermatol. 2006; 45: 139-45

9. Maleki D, Sayyah A, Rahimi-Rad MN et al. Kimura's Disease with eosinophilicpanniculitis treated with cyclosporine: A case report. Allergy Asthma Clin Immunol. 2010; 6: 5

10. Gumbs MA, Pai NB, Sraiya RJ et al. Kimura's Disease: A case report and literature review. J SurgOncol 1999; 70:190-3

11. Itami J, Miyoshi T, Ogata $\mathrm{N}$ et al. Radiation therapy in Kimura's Disease. Acta Oncol. 1989; 82: 549-55

12. Day TA, Abreo F, Aarstad RF et al. Treatment of Kimura's Disease: A therapeutic enigma. Otolaryngology Head Neck surgery $1995 ; 112: 333-7$

13. Li TJ, Chen XM, Wang SZ et al. Kimura's disease: A clinicopathologic study of 54 Chinese patients. Oral Surg Oral Med Oral Pathol Oral Radiol Endod. 1996; 82:549-55. 